\title{
The Global Trend in Sea Surface Temperature from 20 Years of Advanced Very High Resolution Radiometer Data
}

\author{
S. A. Good, G. K. Corlett, J. J. Remedios, E. J. Noyes, And D. T. Llewellyn-Jones \\ Earth Observation Science, Space Research Centre, Department of Physics and Astronomy, University of Leicester, \\ Leicester, United Kingdom
}

(Manuscript received 26 January 2006, in final form 6 July 2006)

\begin{abstract}
The trend in sea surface temperature has been determined from $20 \mathrm{yr}$ of Advanced Very High Resolution Radiometer Pathfinder data (version 5). The data span the period from January 1985 to December 2004, inclusive. The linear trends were calculated to be $0.18^{\circ} \pm 0.04^{\circ}$ and $0.17^{\circ} \pm 0.05^{\circ} \mathrm{C}$ decade $^{-1}$ from daytime and nighttime data, respectively. However, the measured trends were found to be somewhat smaller if version 4.1 of the Pathfinder data was used, or if the time series of data ended earlier. The influence of El Niño on global temperatures can be seen clearly in the data. However, it was not found to affect the trend measurements significantly. Evidence of cool temperatures after the eruption of Mount Pinatubo in 1991 was also observed.
\end{abstract}

\section{Introduction}

The extent of change in the climate due to human influences is currently the subject of much debate. One of the environmental parameters that can be used to track climate change is sea surface temperature (SST; Houghton et al. 2001). Therefore, it is of importance that accurate measurements of trends in SST are obtained, in order to detect climate change and to provide a test bed for models.

If reliable estimates of trends are to be found, datasets covering long time periods are required (Allen et al. 1994). For SST, these exist through in situ observations (e.g., from buoys and voluntary observing ships) and satellite observations. Casey and Cornillon (2001), for example, used SSTs from the World Ocean Atlas 1994 and the Comprehensive Ocean-Atmosphere Data Set (COADS) to determine trends in SSTs since 1942. They found a warming trend of between $0.09^{\circ}$ and $0.14^{\circ} \mathrm{C}$ decade $^{-1}$ (depending on dataset and averaging approach). Houghton et al. (2001) quote a global increase of $0.14^{\circ} \pm 0.04^{\circ} \mathrm{C}$ decade $^{-1}$ between 1976 and

Corresponding author address: Dr. G. K. Corlett, Earth Observation Science, Space Research Centre, Department of Physics and Astronomy, University of Leicester, University Road, Leicester LE1 7RH, United Kingdom.

E-mail: gkc1@leicester.ac.uk

DOI: $10.1175 /$ JCLI4049.1

(C) 2007 American Meteorological Society
2000, from Jones et al. (2001). Studies have also attempted to use satellite data alone to detect trends. Although they span relatively short time periods compared to in situ data, satellite datasets have the advantage that they can provide almost global ocean coverage, allowing global trends to be determined. Datasets made up of in situ observations must utilize a large number of sources to achieve coverage over a wide area and a long time period, each of which has a different bias and sensitivity to observing conditions and might experience drift in their measurements. Only a single satellite instrument is required to achieve almost global coverage of the oceans, and the entire dataset consists of data from only a relatively small number of similar instruments. Although it is true that inconsistencies will exist between these instruments, effort has been put into minimizing these problems, for example, the Pathfinder project (Kilpatrick et al. 2001).

Two global satellite SST datasets currently exist that are long enough for climate change detection. The Advanced Very High Resolution Radiometers (AVHRRs) have been operating on board National Oceanic and Atmospheric Administration (NOAA) polar-orbiting satellites since 1981, providing a continuous source of SSTs. The Along-Track Scanning Radiometer (ATSR) series has spanned three instruments: ATSR-1 and -2 on European Remote Sensing Satellite-1 (ERS-1) and -2, and Advanced ATSR on Envisat, with the data record 
beginning in 1991. Using a 13-yr time series of AVHRR data, Strong et al. (2000) determined that globally averaged SSTs had a positive trend, although the error on the trend was larger than the trend itself. Anderson et al. (2002), utilizing almost $8 \mathrm{yr}$ of ATSR data, identified significant spatial variability in SST trends. They found that the highest increases in SST were situated in the northwest Atlantic, the northwest and southwest Pacific, and in areas to the south of Africa and to the west of Australia. Negative trends were also found in the mid- and northeast Pacific and at the lowest latitudes. Most recently, Lawrence et al. (2004) compared global trends determined from $16 \mathrm{yr}$ of the Pathfinder reprocessing of AVHRR data (version 4.1) and $8 \mathrm{yr}$ of ATSR data. Their methodology included removing the El Niño component from the data, which can reduce the length of time series required to detect trends with confidence by 1-2 yr (Allen et al. 1994). They found consistent trends of $0.09^{\circ} \pm 0.03^{\circ}$ and $0.13^{\circ} \pm 0.06^{\circ} \mathrm{C}$ decade $^{-1}$ from AVHRR and ATSR, respectively. The consistency between the independent datasets indicates that the trends are not significantly biased by instrumental drift but represent a real warming of the ocean surface.

Since Lawrence et al. (2004) published their findings, the AVHRR Pathfinder dataset has reached $20 \mathrm{yr}$, potentially allowing trends to be determined with greater confidence than in the previous studies (Allen et al. 1994). In addition, the dataset has been updated to a new version. In this paper we determine the SST trend from these data and discuss any differences with the previous studies.

\section{Data}

The AVHHR instruments utilized in this paper are a series of space-based, nadir-viewing radiometers that were designed to make measurements in five channels located at selected wavelengths in the visible, nearinfrared, and thermal infrared (see, e.g., Cracknell 1997). The thermal infrared channels, namely, channels $4(10.5-11.5 \mu \mathrm{m})$ and $5(11.5-12.5 \mu \mathrm{m})$, are calibrated through views of a blackbody at around $290 \mathrm{~K}$ and cold space. The spatial resolution of the channels is $4 \mathrm{~km} \times$ $4 \mathrm{~km}$ in global mode with a swath width of approximately $2900 \mathrm{~km}$. One of the applications of its data is to make accurate retrievals of SST from the thermal channels. The Pathfinder SST dataset is a historical reprocessing of AVHRR data (beginning in 1985), which uses a consistent retrieval methodology over all the instruments and attempts to improve the calibration between the instruments in the AVHRR series to provide a high-quality dataset for climate change detection.
Two versions of the Pathfinder dataset are utilized in this study, version 4.1 and version 5 .

The Pathfinder retrieval uses a nonlinear algorithm to calculate SST from the radiances recorded by the instrument. The form of the algorithm is (Walton et al. 1998; Kilpatrick et al. 2001)

$$
\begin{aligned}
\mathrm{SST}_{\mathrm{sat}}= & a+b T_{4}+c\left(T_{4}-T_{5}\right) \mathrm{SST}_{\text {guess }} \\
& +d\left(T_{4}-T_{5}\right)[\sec (\theta)-1] .
\end{aligned}
$$

The algorithm requires radiances recorded in two of the AVHRR channels $\mathrm{T}_{4}$ and $\mathrm{T}_{5}$ (centered at 10.8 and $11.4 \mu \mathrm{m}$ ), a first-guess SST value ( $\mathrm{SST}_{\text {guess }}$ ), the satellite zenith angle $(\theta)$, and a set of four coefficients (a, b, c, and d) in order to calculate SST. The coefficients are determined empirically by regression to in situ SST data from buoys, and for each month the coefficients are calculated by a weighted average over the 5 months centered on that month. There are two periods in the data record that do not follow this approach. First is the period following the eruption of Mount Pinatubo in 1991, which injected large amounts of aerosol into the stratosphere and increased the atmospheric correction required in the retrieval (Kilpatrick et al. 2001). Second, version 4.1 data from January 2000 onward and version 5 data from January 2002 onward are currently interim versions. This means that coefficients from the previous year have been used to retrieve SST. The same coefficients are used for day and night (Kilpatrick et al. 2001). The datasets use data from a number of different AVHRRs on different polar-orbiting satellites; these are NOAA-9 (4 January 1985-7 November 1988), NOAA-11 (8 November 1988-13 September 1994), NOAA-9 (14 September 1994-21 January 1995), NOAA-14 (22 January 1995-11 October 2000), NOAA16 (12 October 2000-31 December 2002), and NOAA17 (1 January 2003-present) (see http://www.nodc. noaa.gov/sog/pathfinder $4 \mathrm{~km} /$ userguide.html).

There are three principal differences between the version 4.1 and version 5 datasets (source: http://www. nodc.noaa.gov/sog/pathfinder $4 \mathrm{~km} /$ userguide.html). These are

(i) improved spatial resolution-version 5 datasets can be obtained on equal-angle grids of size 8196 pixels $\times 4096$ pixels, compared to $4096 \times 2048$ pixels for version 4.1 data;

(ii) improved ice mask identification-the Special Sensor Microwave Imager (SSM/I) and the Reynolds optimal interpolation SST dataset are used to identify regions containing ice; and

(iii) improved land mask - a new 1-km resolution land mask developed from Moderate Resolution Imaging Spectroradiometer (MODIS) data is used for 
version 5 data, compared to a 9-km mask used for version 4.1. This is then degraded to the resolution of the version 5 SST product.

AVHRR Pathfinder SST data (version 4.1 and version 5) were downloaded from the Physical Oceanography Distributed Active Archive Center (PO.DAAC; http://podaac.jpl.nasa.gov) and National Oceanographic Data Center (NODC; http://www.nodc.noaa. gov). These datasets are monthly averages, with the former on a 54-km equal-angle grid and the latter at 4-km spatial resolution.

\section{Methodology}

While time series of SST anomalies show much shortterm natural variability, it is the goal of studies such as these to make an assessment of the longer-term changes in temperature over the period of data coverage. A number of studies have applied trend models to surface or air temperature data, concluding that linear trend and breakpoint models are appropriate to global analyses, for example, Zheng and Basher (1999) and Siedel and Lanzante (2004), rather than random walk models with no deterministic trends as suggested as potentially plausible by Woodward and Gray (1993). Siedel and Lanzante (2004) performed a detailed study employing flat-step models, piecewise linear models, sloped-step models, and linear models with fits to a number of datasets. Where abrupt changes could be identified in datasets, for example, in 1946 and 1977, multipart models were preferred. However, in both the portion of the surface temperature record beyond 1977 and in fits to 1979-2001 tropospheric temperature datasets from satellites and radiosondes, no such abrupt changes could be identified and linear trends were deduced. In the case of the 1979-2001 datasets, a linear trend with the first-order autoregressive [AR(1)] model was found to best explain data records. Therefore, this is the model that is adopted for our analysis.

Our methodology is equivalent to that of Lawrence et al. (2004) in that the seasonal cycle and the influence of El Niño are removed from the SST datasets, allowing the trend due to other natural processes and anthropogenic influences to be determined. However, rather than performing these steps separately as in Lawrence et al. (2004) we adapt the formulism of Weatherhead et al. (1998) to find the seasonal cycle, influence of El Niño, and trend simultaneously, and then to calculate the error on the trend. In brief, the analysis consists of the following steps.

We adopt the criterion of Lawrence et al. (2004) of requiring continuous data at a spatial point throughout the time series if they are to be included in the analysis. This avoids the possibility of trends being biased by a lack of data points. Therefore, the data are first averaged to $2.5^{\circ}$ spatial resolution in order to minimize any data gaps due to cloud and to achieve near-global coverage. Only data flagged as having the highest quality in the Pathfinder auxiliary information (flag value of seven) were used. Any grid cells that did not have continuous data throughout the time series are ignored in the following analysis.

The strength of El Niño during each month of the time series is then determined as in Lawrence et al. (2004). An empirical orthogonal function (EOF) analysis is performed on the region of the eastern Pacific cold tongue $\left(10^{\circ} \mathrm{S}-10^{\circ} \mathrm{N}, 150^{\circ}-75^{\circ} \mathrm{W}\right)$ after first removing the seasonal cycle at each spatial point (found by averaging the data for each January together, each February together, etc.). The disappearance of the cold tongue is one of the characteristics of an El Niño event, and hence changes in SST in this region can be used to infer the occurrence and strength of El Niño. After the seasonal cycle, the dominant mode of variability in the region (represented by the first EOF) is the change in SST due to El Niño. The corresponding principal component (PC) time series, obtained by projecting the EOF back onto the data, then provides a function representing the strength of El Niño.

A model containing the expected variability within the SST datasets is then fitted to a time series of globally averaged SSTs using a nonlinear least squares fitting function that is part of Interactive Data Language (IDL). The global averages are produced by finding the mean of all the $2.5^{\circ} \times 2.5^{\circ}$ grid cells, weighted by the surface area of the earth represented by each cell. The model (adapted from Weatherhead et al. 1998) is

$$
T_{t}=\mu+S_{t}+\frac{1}{12} \omega t+\alpha \mathrm{PC}_{t},
$$

where global-average SST (T) at time $t$ (in months) is described by a constant term $(\mu)$, a seasonal component $\left(S_{t}\right)$, a linear trend of rate $\omega^{\circ} \mathrm{C}$ year ${ }^{-1}$, and a constant $(\alpha)$ multiplied by the function $\mathrm{PC}_{t}$ obtained previously that represents the strength of El Niño. The seasonal component is represented by Weatherhead et al. (1998)

$$
S_{t}=\sum_{j=1}^{4}\left[\beta_{1, j} \sin \left(\frac{2 \pi j t}{12}\right)+\beta_{2, j} \cos \left(\frac{2 \pi j t}{12}\right)\right],
$$

with the values of the $\beta$ terms determined during the fit.

The residual variability that has not been explained by the model is assumed to be autoregressive of order 1 (Weatherhead et al. 1998):

$$
R_{t}=\phi R_{t-1}+\epsilon_{t},
$$



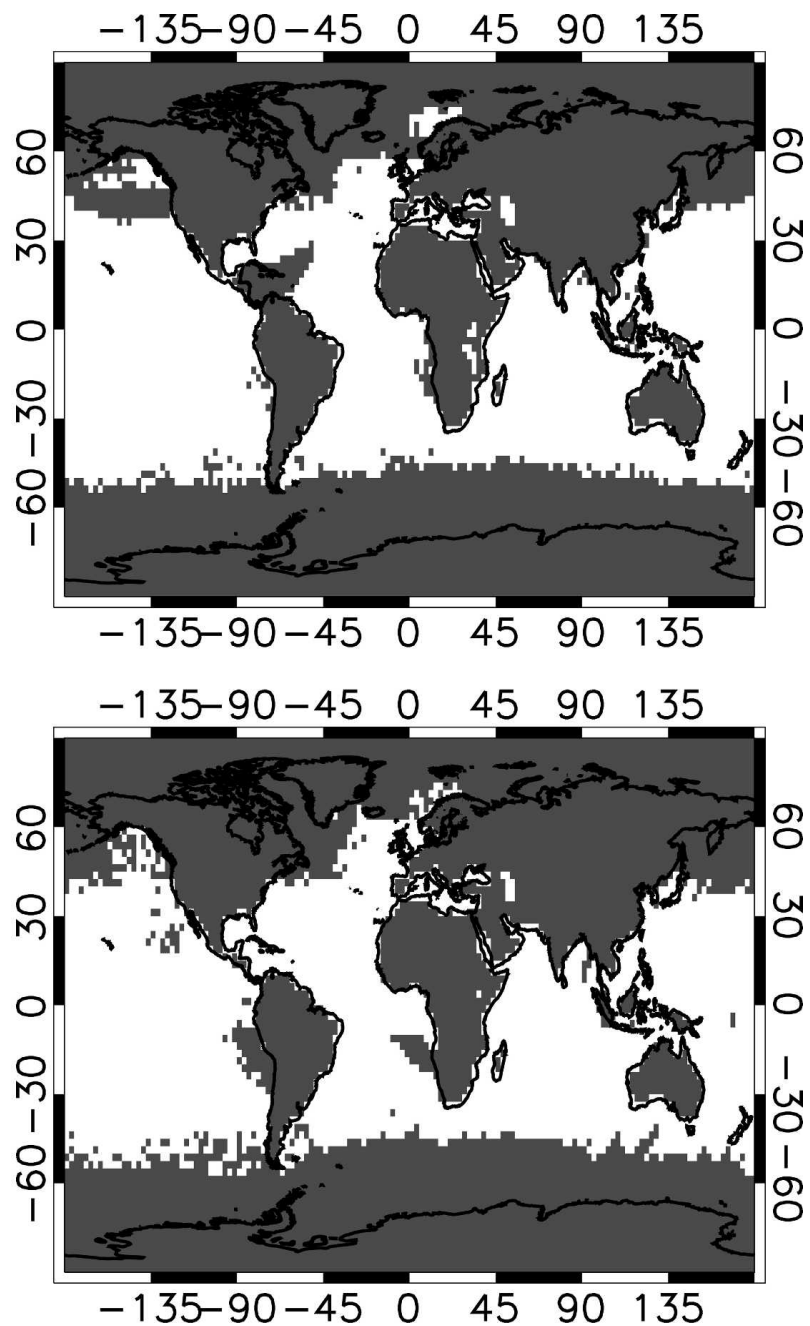

FIG. 1. Coverage of the globe under the criterion that there must be continuous data in a location for them to be included in the analysis. White denotes where this criterion is fulfilled, gray where there is land or where cloud has prevented measurements during a month. (top) Day and (bottom) night.

where the factor $\phi$ allows the residuals $(R)$ at time $t$ to be dependent on the residuals at $t-1$, which will be the case if the variability is not random and persists over time. The remaining variability $\epsilon_{t}$ is assumed to be random with variance $\sigma_{\epsilon}^{2}$. The factor $\phi$ is determined by fitting a model $R_{t}=\phi R_{t-1}$ to the residuals to obtain $\phi$; then $\sigma_{\epsilon}$ is found by calculating the standard deviation of any variability still not explained. The error on the trend estimate is then given by (Weatherhead et al. 1998)

$$
\sigma_{\omega} \approx \frac{\sigma_{\epsilon}}{(1-\phi) n^{3 / 2}},
$$

where $\sigma_{\omega}$ is the standard deviation of the trend estimate and $n$ is the number of years of data.

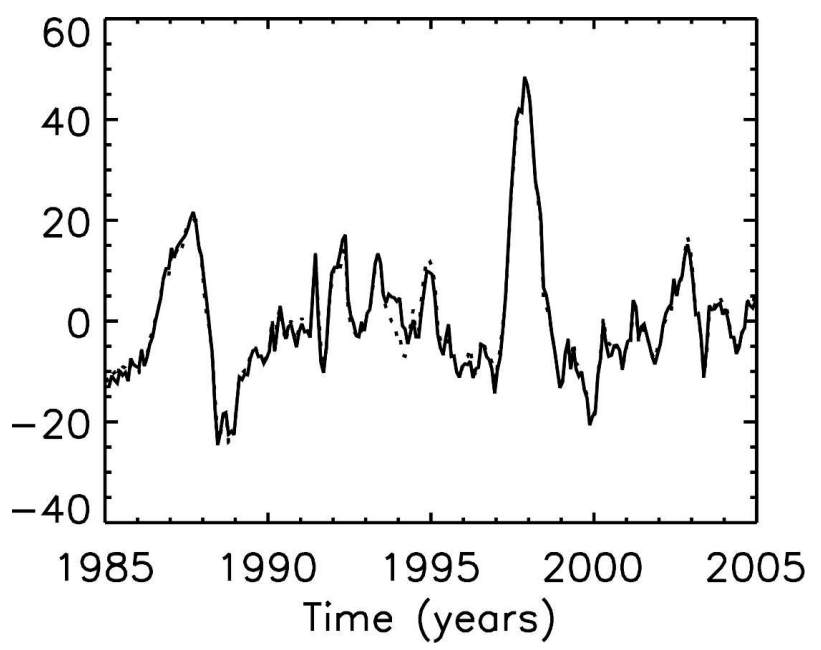

FIG. 2. PCs corresponding to the strength of El Niño obtained from EOF analysis (solid line: day, dotted: night).

\section{Results}

Figure 1 shows the coverage of the globe when $2.5^{\circ}$ spatial averaging and the criterion for continuous data coverage is applied. There are some notable gaps in the data coverage, for example, in the western Atlantic and northern Pacific Oceans in the daytime data and off the west coasts of South America and Africa in the nighttime image. The effect of these missing spatial points is discussed in section 5. While the averages used in this work are described as "global," in practice it can be seen that because of the strict criterion of having continuous data throughout the time series, the coverage toward the poles is relatively poor. Overall the global averages consist of temperatures from $72 \%$ of the World Ocean for both day and night. Increasing this percentage could be achieved by relaxing the continuous coverage criterion, or by performing some kind of interpolation to fill in data cells that do not have data. We have not attempted either of these since, as discussed in Lawrence et al. (2004), these methods could potentially introduce biases into the trends.

The PCs corresponding to the strength of El Niño, obtained from the EOF analysis, are shown in Fig. 2. The correlation between the day and night PCs is very high $(r=0.99)$. The PCs also have high correlations with the Southern Oscillation index $(r=-0.62$; data obtained from http://www.cgd.ucar.edu/cas/catalg/ climind/soi.html; see Trenberth 1984) and the Multivariate El Niño-Southern Oscillation index $(r=0.89$; data obtained from http://www.cdc.noaa.gov/people/ klaus.wolter/MEI/mei.html; see Wolter and Timlin 1993, 1998), suggesting that this EOF approach is valid. In addition, clear peaks can be seen in the PCs around 
TABLE 1. Values of the model parameters that best allow the model to reproduce the data.

\begin{tabular}{lrr}
\hline \hline & \multicolumn{1}{c}{ Day } & Night \\
\hline$\mu\left({ }^{\circ} \mathrm{C}\right)$ & 295.793 & 295.654 \\
$\beta_{1,1}$ & 0.254 & 0.204 \\
$\beta_{2,1}$ & 0.105 & 0.002 \\
$\beta_{1,2}$ & 0.053 & 0.035 \\
$\beta_{2,2}$ & -0.055 & -0.039 \\
$\beta_{1,3}$ & 0.002 & 0.001 \\
$\beta_{2,3}$ & -0.002 & 0.005 \\
$\beta_{1,4}$ & 0.001 & 0.003 \\
$\beta_{2,4}$ & -0.001 & 0.000 \\
$\alpha$ & 0.005 & 0.005 \\
$\omega\left({ }^{\circ} \mathrm{C} \mathrm{yr}{ }^{-1}\right)$ & 0.018 & 0.017 \\
$\sigma_{\omega}\left({ }^{\circ} \mathrm{C} \mathrm{yr}{ }^{-1}\right)$ & 0.004 & 0.005 \\
$\phi$ & 0.843 & 0.860 \\
$\sigma_{\epsilon}\left({ }^{\circ} \mathrm{C}\right)$ & 0.056 & 0.058 \\
\hline
\end{tabular}

1987 and 1997, when strong El Niño events occurred. Evidence for weaker events can also be seen, for example, in 1992, 1993, and 1995.

The values of the parameters that allow the model to best reproduce the data are given in Table 1 . As might be expected, the constant term $(\mu)$ is warmer for the daytime data than for the night, by $0.139 \mathrm{~K}$. The values of the $\beta$ terms describe the seasonal cycle found by the fit. Correlation of the seasonal cycles determined using this method with those obtained by averaging the data for each month (as used by Lawrence et al. 2004) yields very high correlation coefficients (0.9998 and 0.9997), suggesting that the methods are consistent and that the parameterization used in the model is valid.

Figures 3 and 4 show the data with the model overplotted. The center panels show the SST anomalies resulting from subtracting only the seasonal cycle component of the model from the data. The lower panels in the plots show the difference between the data and the model. The effect of the El Niño component of the model can be seen clearly in the increased temperatures during the strong El Niño years of 1987 and 1997. There is some evidence that the global effect of El Niño continues longer than determined from the EOF analysis, from the high anomalies and residuals immediately after the 1987 and 1997 events. Indeed, Lawrence et al. (2004) identified global El Niño effects related to the 1997 event with a time lag of 14 months. The linear trends determined by the fits are $0.18^{\circ}$ and $0.17^{\circ} \mathrm{C}$ decade $^{-1}$ for day and night, respectively. If the El Niño component of the model is not included, these are both increased by $0.01^{\circ} \mathrm{C}$. Therefore, El Niño only has a weak influence on the measured trends, despite being the strongest mode of natural variability in the data, after the seasonal cycle.

The residuals of the fit show considerable structure.

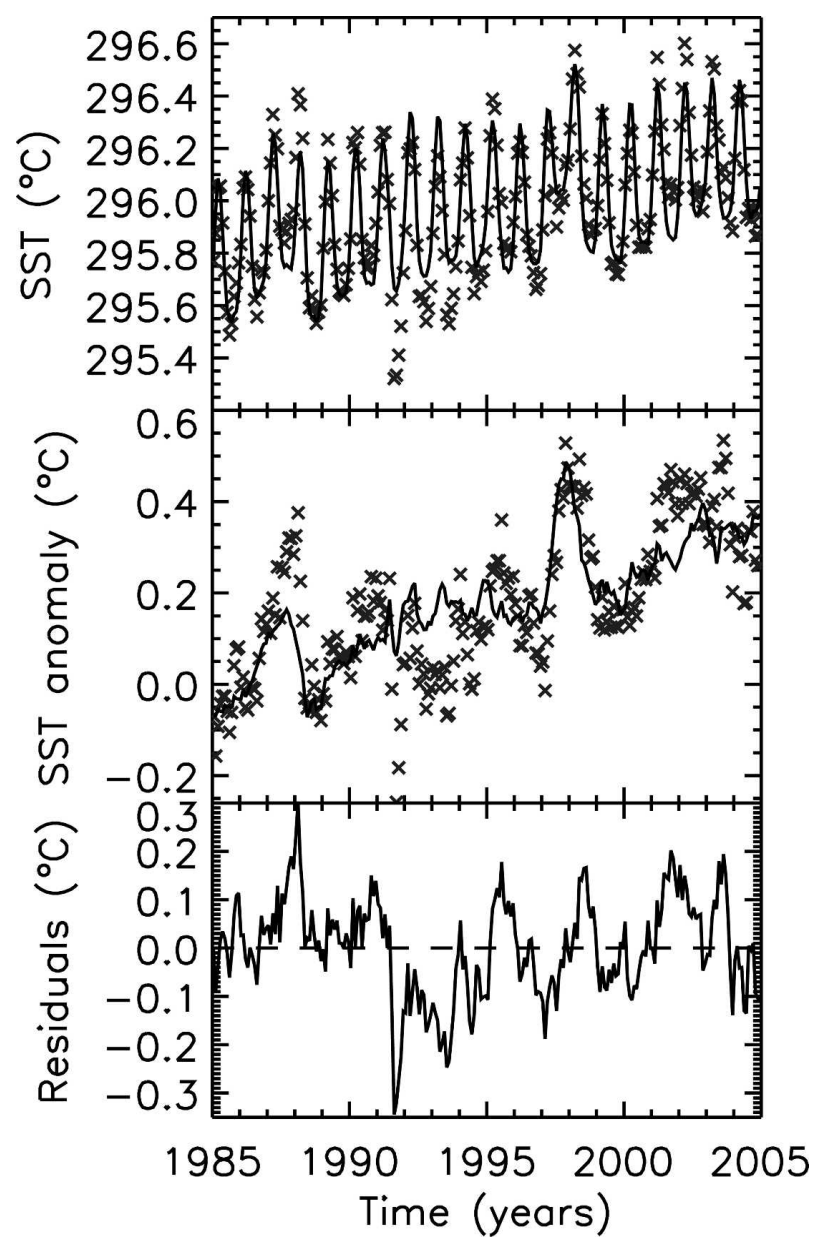

FIG. 3. (top) Globally averaged daytime SSTs (crosses) and fitted model (solid line), (center) the anomalies after subtraction of the seasonal component of the model, and (bottom) the differences between data and the model (dashed line indicates zero residuals).

For example, there are negative residuals between 1991 and 1995. These are likely to be associated with cooling due to the ejection of aerosols into the stratosphere by the Mount Pinatubo eruption of 1991. Lawrence et al. (2004) investigated these cool anomalies using in situ data. They concluded that the satellite instruments were recording a real cooling of the surface during this time, rather than the low temperatures being due to a retrieval bias. The structure in the residuals is reflected in a histogram of the values, which shows clear differences from the best-fitting Gaussian distribution (Fig. 5), and in the high values of $\phi(0.843$ and 0.860 for day and night, respectively). This suggests that over the time series the residuals in a month are well correlated with the residuals in the previous month. After removal of the correlated portion of the residuals, the histogram of the data is very close to Gaussian (Fig. 6), indicating 


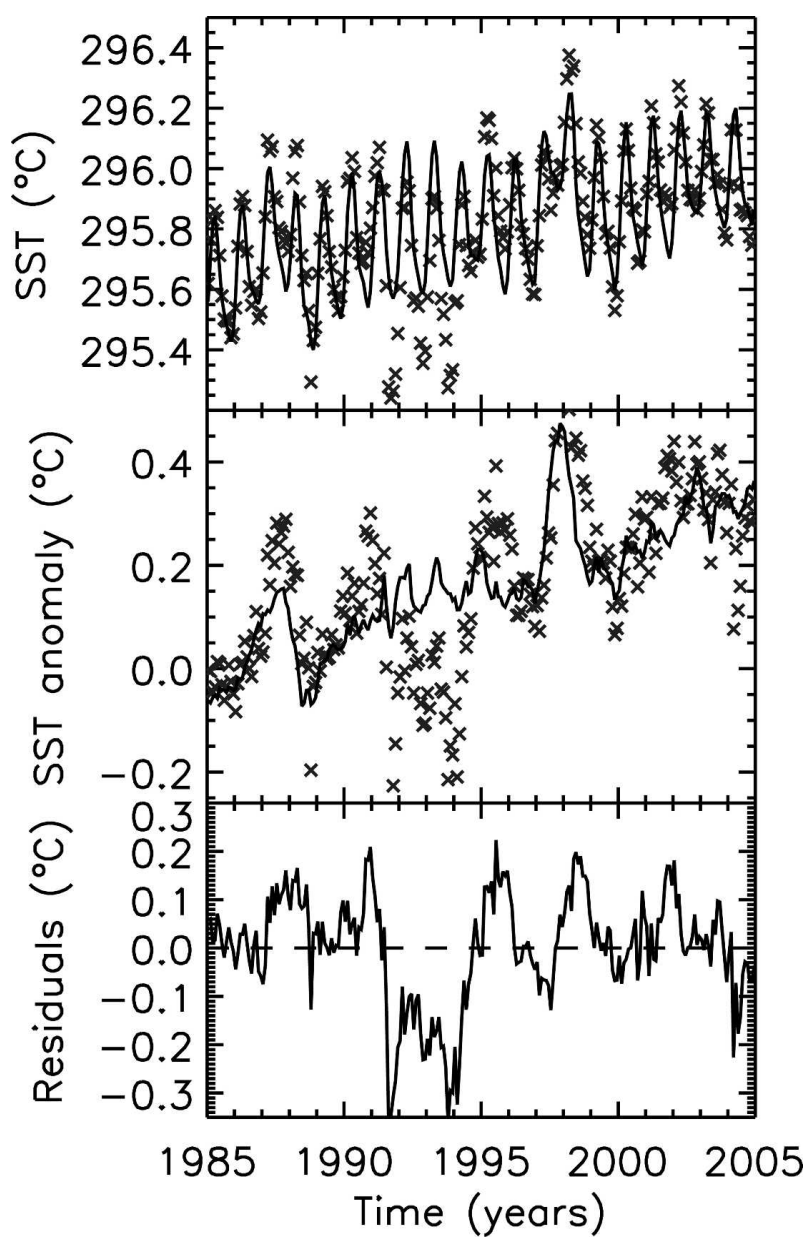

FIG. 4. Same as in Fig. 3 but showing nighttime data and the fitted model.

that the assumption of a first-order autoregressive model for the residuals was good. The random component of the residuals model was calculated to have standard deviations of $0.056^{\circ}$ and $0.058^{\circ} \mathrm{C}$ for day and night, respectively.

From the above values, the standard deviations of the measured trends were determined to be $0.04^{\circ}$ and $0.05^{\circ} \mathrm{C}$ decade $^{-1}$ for the daytime and nighttime datasets, respectively. Therefore, the trends calculated from SSTs retrieved from daytime and nighttime data are consistent to within error and demonstrate with $>99.9 \%$ certainty that SSTs have increased globally over the past two decades. However, it should be noted that the values of $\phi$ also have an uncertainty associated with them, which in turn causes some uncertainty in the standard deviations of the trends. Adopting the standard deviations of the random variability in the residuals calculated above, the $1 \sigma$ error on the values of $\phi$ determined in the fit were found to be 0.035 for daytime data and 0.033 for nighttime data. The corresponding

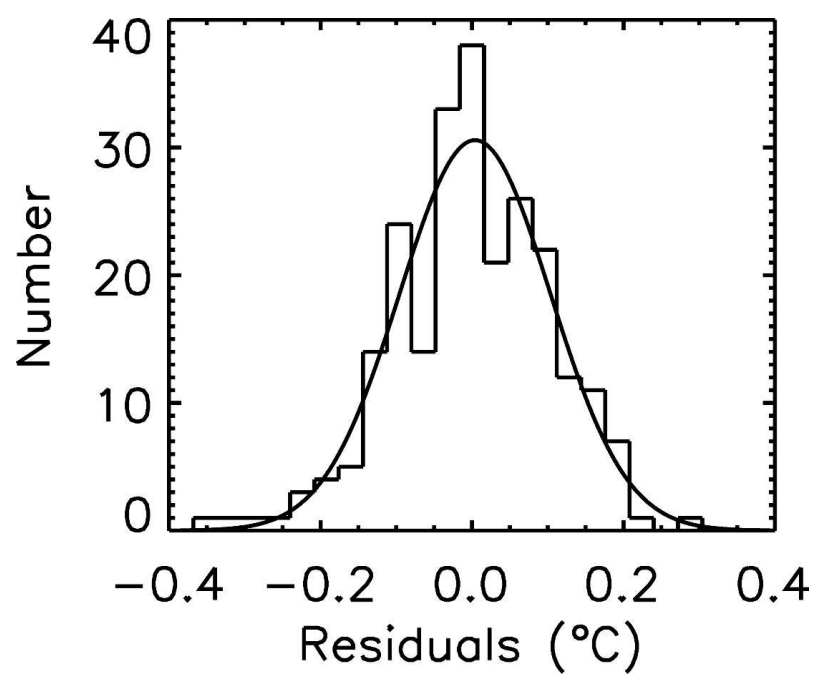

FIG. 5. Histogram of the residual variability remaining after subtraction of the model from the global average SSTs for daytime data with the best-fitting Gaussian distribution overplotted.

change in the calculated standard deviation of the trends if the value of $\phi$ is under- or overestimated by 1 $\sigma$ is an increase or decrease of $0.01^{\circ} \mathrm{C} \mathrm{decade}^{-1}$, respectively (day and night). Neglecting the El Niño component of the model also changes the standard deviations of the trends, increasing them by $0.01{ }^{\circ} \mathrm{C}$ decade $^{-1}$. Hence, removing natural variability such as that due to El Niño increases the confidence in the trend.

\section{Discussion}

This study has determined that SSTs are warming by $0.18^{\circ} \pm 0.04^{\circ}$ and $0.17^{\circ} \pm 0.05^{\circ} \mathrm{C}$ decade $^{-1}$ globally,

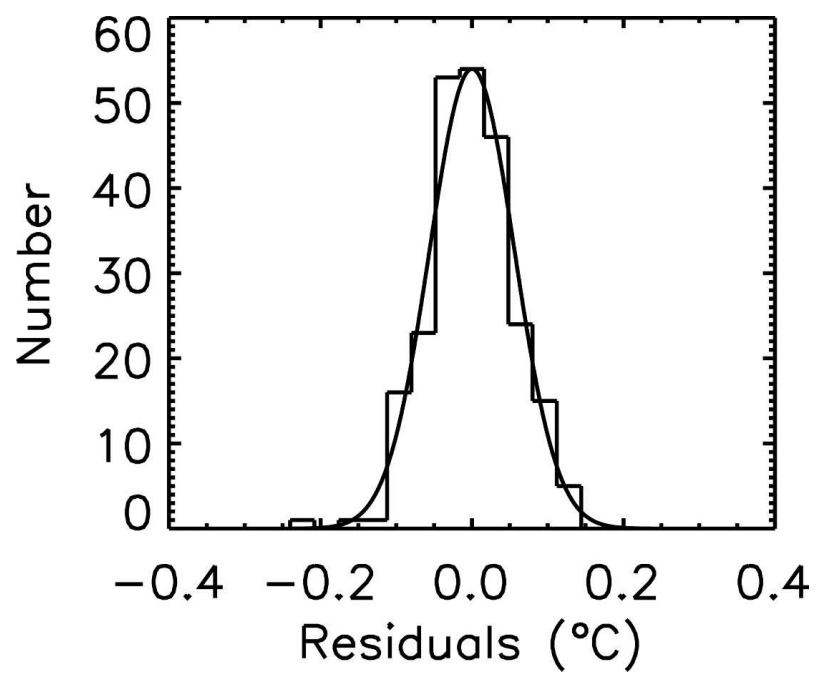

FIG. 6. Histogram of the residual variability after the autocorrelated portion has been removed. The overplotted curve shows a Gaussian distribution with standard deviation $\sigma_{\epsilon}$ 
from daytime and nighttime AVHRR Pathfinder version 5 data, respectively. This trend is consistent, within errors, to those determined by Casey and Cornillon (2001), Houghton et al. (2001), and Lawrence et al. (2004), where ATSR data were used. However, the most comparable trend to those found in this paper is that determined by Lawrence et al. (2004) using AVHRR Pathfinder daytime data. They found the SST trend to be $0.09^{\circ} \pm 0.03^{\circ} \mathrm{C}$ decade $^{-1}$. The two trends are different by over twice the error on our trend determination (three times if their error is used), despite the data source being the same. Here we investigate the possible reasons for this:

(i) Lawrence et al. (2004) looked at a shorter time span of data, spanning between 1985 and 2001. Restricting our analysis to the time period they used decreases our daytime trend to $0.13^{\circ} \pm$ $0.05^{\circ} \mathrm{C}$ decade $^{-1}$, giving much better agreement with the results of Lawrence et al. (2004).

(ii) Lawrence et al. (2004) performed their analysis slightly differently, first removing the seasonal cycle and El Niño, then determining a trend from the resultant anomalies. Retaining the shortened time period and performing the analysis in this way also results in a trend of $0.13^{\circ} \mathrm{C}$ decade $^{-1}$, suggesting that the two analysis procedures are equivalent. If the Lawrence et al. (2004) method of calculating error and their estimate of the error on each global average $\left(0.16^{\circ} \mathrm{C}\right)$ are assumed, the error on the trend is found to be $\pm 0.03^{\circ} \mathrm{C}$ decade $^{-1}$. However, this error estimate does not attempt to take into account the autocorrelation of the residuals.

(iii) Lawrence et al. (2004) used an older version of Pathfinder dataset than that utilized in this study. The trend determined here by performing our analysis on Pathfinder version 4.1 data over the restricted time period was $0.09^{\circ} \pm 0.04^{\circ} \mathrm{C}$ decade $^{-1}$. This trend is identical to that found by Lawrence et al. (2004) with AVHRR version 4.1 data, again confirming the equivalence of the analysis methods. Therefore, changing the version of the Pathfinder data that is used has changed the measured trend by $0.04^{\circ} \mathrm{C}$ decade $^{-1}$.

Enhancements that were introduced into version 5 of the Pathfinder dataset compared to version 4.1 are improved spatial resolution, ice mask identification, and land mask (source: http://www.nodc.noaa.gov/sog/ pathfinder $4 \mathrm{~km} /$ userguide.html). The newer dataset may therefore include data closer to land. It is also possible that trends determined from the older dataset were biased by the incorrect inclusion of ice or land

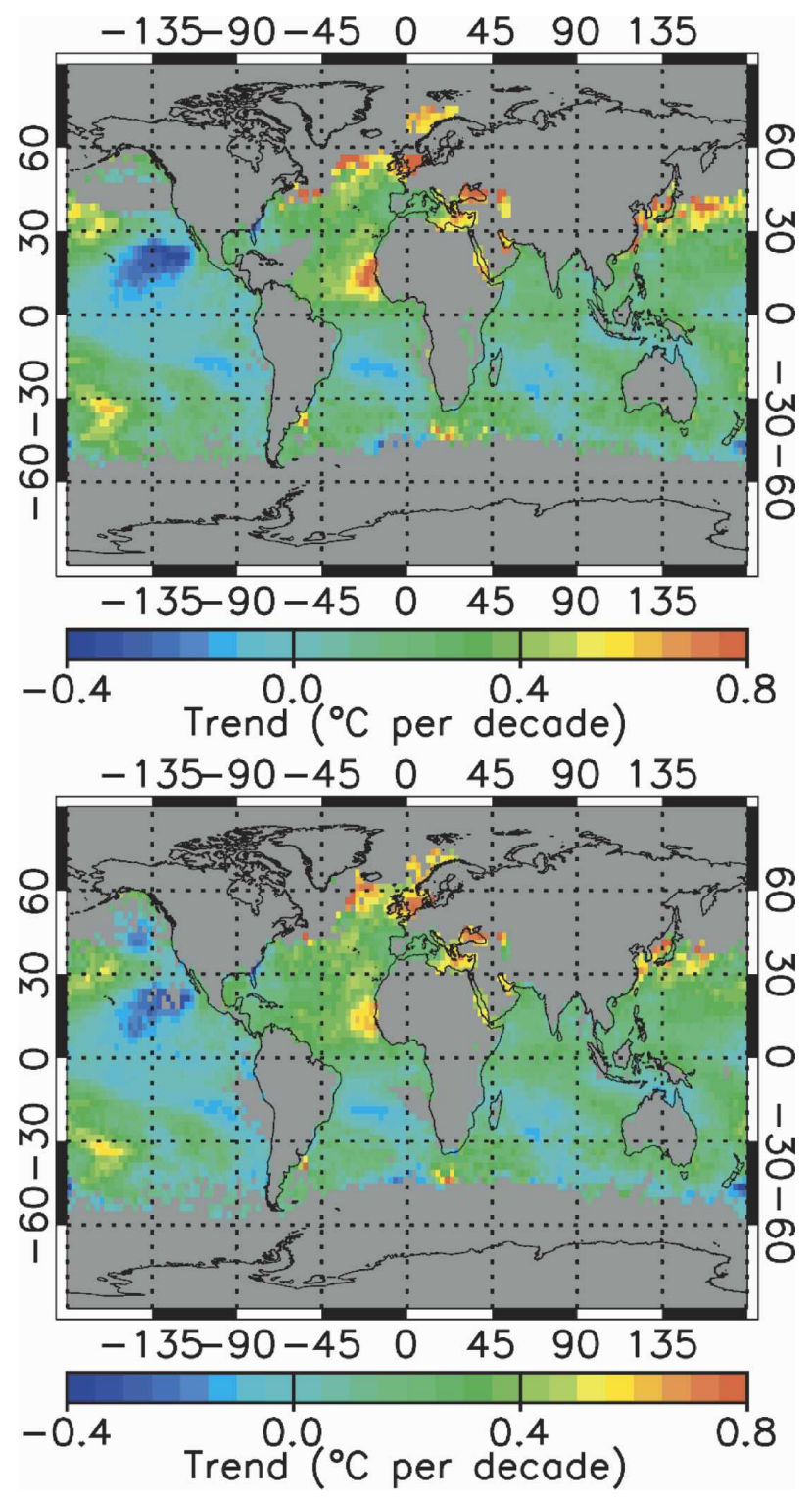

FIG. 7. Spatial pattern of trends in SST from (top) daytime and (bottom) nighttime AVHRR Pathfinder version 5 data over the period from January 1985 to December 2004.

pixels or the exclusion of sea pixels. In addition, since climate trends are not uniform over the globe, but show significant regional differences, the difference in masks that mark where data gaps due to cloud and land lie in the datasets could affect the trends. Therefore, this could be the reason, or part of the reason, for the differences that have been identified between version 4.1 and version 5 Pathfinder trends. The spatial pattern of trends was determined by performing our analysis on the data for each grid cell individually, rather than on the global averages. Figure 7 shows the resulting trends from the day and night data over the full time period of 


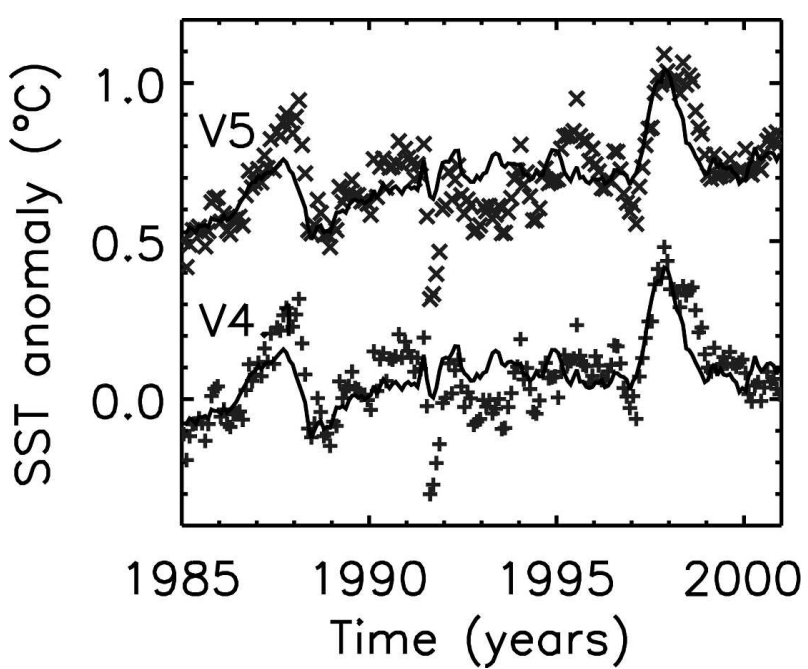

FIG. 8. Comparison of the time series of the averaged SSTs with the seasonal cycle removed from version 4.1 (+ symbols) and 5.0 $(\times)$ daytime datasets, with the models overlaid. The version 5.0 data have been offset vertically for clarity. To provide a direct comparison, the averages are formed only from grid cells where there are data throughout both versions of the data.

Pathfinder version 5 data. These patterns seem consistent with those observed by Strong et al. (2000) and Anderson et al. (2002). In the spatial maps, high warming trends $\left(>0.5^{\circ} \mathrm{C}\right.$ decade $\left.^{-1}\right)$ are observed at the northern limits of the coverage, as well as off the western coast of Africa. Negative trends are also seen, for example, in the northeastern Pacific and in the southern oceans.

It was noted in section 4 that the cloud and land masks for the daytime and nighttime data differed, although the measured climate trends were not significantly different. To determine if the trend measured from version 4.1 data is being affected by the differing cloud and land masks, a consistent mask for both datasets was determined; that is, a mask was produced that marked where continuous data were present in both version 4.1 and version 5 datasets over the time period analyzed by Lawrence et al. (2004). Figure 8 shows the resulting times series of SST anomalies for the two versions of the data after removal of the seasonal cycle. While similar features can be seen in the datasets, the anomalies are noticeably higher toward the end of the time series in the version 5 data. This is particularly obvious at the beginning of 1996 and the end of 2000. The trends determined in the analysis for the shortened time period, $1985-2001$, were $0.09^{\circ} \pm$ $0.05^{\circ} \mathrm{C}$ decade $^{-1}$ for daytime version 4.1 data and $0.13^{\circ}$ $\pm 0.05^{\circ} \mathrm{C}$ decade $^{-1}$ for daytime version 5 data. These trends are identical to those determined when differing masks were used.

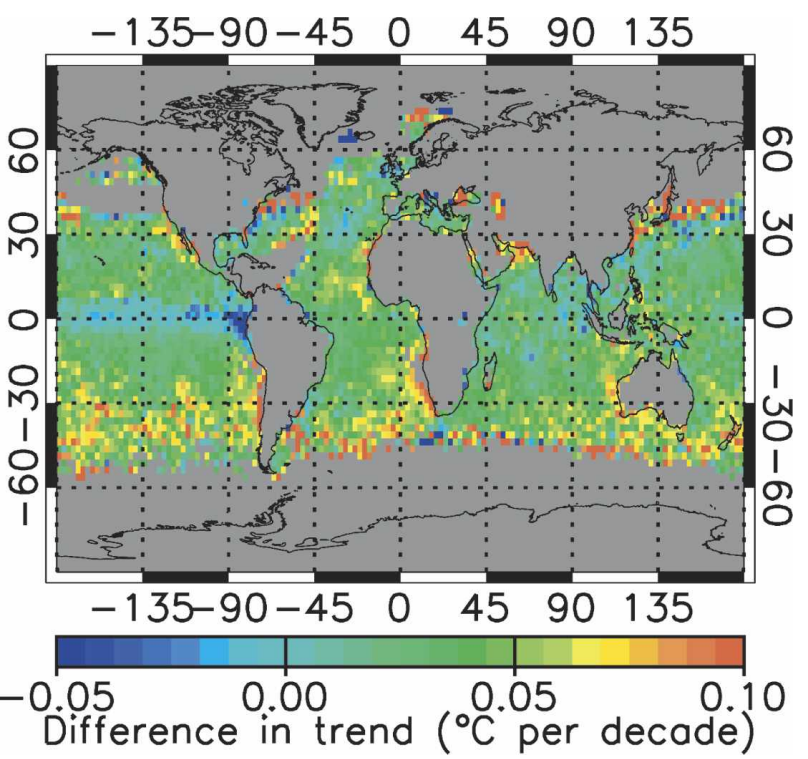

FIG. 9. Differences between the SST trends measured using version 5 and version 4.1 daytime data at each spatial grid point. The differences shown are version 5 - version 4.1 trends.

Figure 9 shows the difference in trend in each grid cell between the two versions of the data (version 5 minus version 4.1). In the plot, the cyan colors indicate no difference in the measured trend. Negative differences in the trends (colored blue) can be seen in the El Niño region. The highest positive differences (yellow and red colors) are seen around the land and toward the most northern and southern parts of the data coverage, where the occurrence of cloud and ice may have prevented there being continuous data. These differences may therefore be related to the changes in land and ice maps. However, green colors, representing smaller positive differences, are observed almost everywhere else. Therefore, the difference in the globally averaged SST trends between the two versions of the datasets cannot be attributed solely to a particular region of the globe.

The error on the trend measured with the newer dataset has also increased by $0.01^{\circ} \mathrm{C}$ decade $^{-1}$. This is due to the value of $\phi$ being larger for the version 5 dataset than for version 4.1 (0.840 compared to 0.789), while the standard deviation of the random component of the residuals model is $0.054^{\circ} \mathrm{C}$ for version 5 and $0.050^{\circ} \mathrm{C}$ for version 4.1 . This indicates that the changes that were made to the Pathfinder processing between version 4.1 and version 5 has included or excluded SSTs, or changed the retrieved temperatures, in such a way that a greater autocorrelation is seen in the residuals. This demonstrates that while version 5 of the Pathfinder dataset can be thought of as an improvement 
over version 4.1, this does not necessarily mean that a trend can be determined to greater precision.

It is therefore possible to conclude that the change in data between versions of the Pathfinder datasets have resulted in an increase in the measured trend. However, this difference is less than one standard deviation in the measured trend and does not account for the full difference between the Lawrence et al. (2004) trend and that found in this paper. Extending the datasets beyond the time period used by Lawrence et al. (2004) also results in an increase in the measured trend; this is true of version 4.1 data as well as the version 5 datasetwhen a time span of version 4.1 data of January 1985 to June 2003 is used, the trend is increased to $0.13^{\circ} \pm$ $0.04^{\circ} \mathrm{C}$ decade $^{-1}$. Therefore, the later part of the time period appears to be increasing the measured trend. However, within errors, the trends determined from the two time periods agree. Data extending the record beyond 2004 are required in order to understand these differences fully and to investigate the changes that occur in the 2001-04 period.

\section{Conclusions}

The AVHRR Pathfinder dataset (version 5) now extends to $20 \mathrm{yr}$ of continuous data. These span the period from January 1985 to December 2004, inclusive, and have been used to study the global trend in SST over that time period. The data, which were obtained as monthly averages, were further averaged to $2.5^{\circ}$ resolution, and any spatial points without continuous data were discarded to avoid biasing the trend measurements. A model consisting of a constant term, a seasonal cycle, an El Niño component, and a linear trend was fitted to global averages of the SSTs. The trend in SST was found to be $0.18^{\circ} \pm 0.04^{\circ}$ and $0.17^{\circ} \pm 0.05^{\circ} \mathrm{C}$ decade $^{-1}$ from daytime and nighttime data, respectively. The influence of El Niño on the trend was found to be weak compared to the error on the trend measurement; when the El Niño component of the model was removed, the measured trends increased by only $0.01^{\circ} \mathrm{C}$ decade $^{-1}$. However, the standard deviation of the trend also increased by $0.01^{\circ} \mathrm{C}$ decade $^{-1}$. Evidence of cool temperatures after the eruption of Mount Pinatubo in 1991 ejected aerosol into the stratosphere was also seen.

The trends determined in this paper are higher than that obtained by Lawrence et al. (2004) when using AVHRR Pathfinder data. The differences can be attributed to changes in the data between version 4.1 and version 5 of the Pathfinder dataset and to the time period over which the trend was measured. The former resulted in a difference of $0.04^{\circ} \mathrm{C}$ decade $^{-1}$ in the trend, while ending the time series in December 2000 resulted in a trend that was $0.05^{\circ} \mathrm{C}$ decade ${ }^{-1}$ less. The difference resulting from the extension of the time series is of similar magnitude to the error on the trend. As the data record extends over the forthcoming years it will be possible to determine if these most recent years are anomalous, resulting in a high trend, or if the trend determined from the shorter dataset was low. In conclusion, these results demonstrate with high certainty that SSTs have increased globally over the past two decades, with an indication of an increased rise in recent years.

Acknowledgments. The authors thank the two referees for their interesting and useful comments. The AVHRR Oceans Pathfinder SST data were obtained from the Physical Oceanography Distributed Active Archive Center (PO.DAAC) at the NASA Jet Propulsion Laboratory, Pasadena, California (http://podaac. jpl.nasa.gov), and from the National Oceanographic Data Center (NODC; http://www.nodc.noaa.gov).

\section{REFERENCES}

Allen, M., C. Mutlow, G. Blumberg, J. Christy, R. McNider, and D. Llewellyn-Jones, 1994: Global change detection. Nature, 370, 24-25.

Anderson, O., P. Knudsen, and B. Beckley, 2002: Monitoring sea level and sea surface temperature trends from ERS satellites. Phys. Chem. Earth, 27, 1413-1417.

Casey, K. S., and P. Cornillon, 2001: Global and regional sea surface temperature trends. J. Climate, 14, 3801-3818.

Cracknell, A. P., 1997: The Advanced Very High Resolution Radiometer. Taylor and Francis, $534 \mathrm{pp}$.

Houghton, J. T., Y. Ding, D. J. Griggs, M. Noguer, P. J. van der Linden, X. Dai, K. Maskell, and C. A. Johnson, Eds., 2001: Climate Change 2001: The Scientific Basis. Cambridge University Press, $944 \mathrm{pp}$.

Jones, P. D., T. J. Osborn, K. R. Briffa, C. K. Folland, E. B. Horton, L. V. Alexander, D. E. Parker, and N. A. Rayner, 2001: Adjusting for sampling density in grid box land and ocean surface temperature time series. J. Geophys. Res., 106, 33713380.

Kilpatrick, K. A., G. P. Podestá, and R. Evans, 2001: Overview of the NOAA/NASA Advanced Very High Resolution Radiometer Pathfinder algorithm for sea surface temperature and associated matchup database. J. Geophys. Res., 106, 91799198.

Lawrence, S. P., D. T. Llewellyn-Jones, and S. J. Smith, 2004: The measurement of climate change using data from the Advanced Very High Resolution and Along Track Scanning Radiometers. J. Geophys. Res., 109, C08017, doi:10.1029/ 2003JC002104.

Siedel, D. J., and J. R. Lanzante, 2004: An assessment of three alternatives to linear trends for characterizing global atmospheric temperature changes. J. Geophys. Res., 109, D14108, doi:10.1029/2003JD004414.

Strong, A. E., E. J. Kearns, and K. K. Gjovig, 2000: Sea surface 
temperature signals from satellites-An update. Geophys. Res. Lett., 27, 1667-1670.

Trenberth, K. E., 1984: Signal versus noise in the Southern Oscillation. Mon. Wea. Rev., 112, 326-332.

Walton, C. C., W. G. Pichel, J. F. Sapper, and D. A. May, 1998: The development and operational application of nonlinear algorithms for the measurement of sea surface temperatures with the NOAA polar-orbiting environmental satellites. $J$. Geophys. Res., 103, 27 999-28 012.

Weatherhead, E. C., and Coauthors, 1998: Factors affecting the detection of trends: Statistical considerations and applications to environmental data. J. Geophys. Res., 103, 17149 17161.
Wolter, K., and M. S. Timlin, 1993: Monitoring ENSO in COADS with a seasonally adjusted principal component index. Proc. 17th Climate Diagnostics Workshop, Norman, OK, NOAA/ NMC/CAC, NSSL, Oklahoma Climate Survey, CIMMS and the School of Meteorology, University of Oklahoma, 52-57. —, and 1998: Measuring the strength of ENSO-How does 1997/98 rank? Weather, 53, 315-324.

Woodward, W. A., and H. L. Gray, 1993: Global warming and the problem of testing for trend in time series data. J. Climate, $\mathbf{6}$, 953-962.

Zheng, X., and R. E. Basher, 1999: Structural time series models and trend detection in global and regional temperature series. J. Climate, 12, 2347-2358. 